\title{
A MULTIPLICATION IN $N$-SPACE
}

\author{
DEAN LUCAS
}

\begin{abstract}
A multiplication is constructed which extends the vector space structure of Euclidean $n$-space to a commutative algebra.
\end{abstract}

1. Introduction. An old problem in mathematics is to endow Euclidean $n$-space with a multiplicative structure so that it becomes a real algebra with decent properties. Among the properties that might be characterized as "decent" are the following:

A. Associativity,

B. Commutativity,

C. Existence of multiplicative inverses or, at least,

$C^{\prime}$. No zero divisors,

D. $|x y| \leqslant|x||y|$ or, failing that,

$\mathrm{D}^{\prime}$. Multiplication is continuous in the Euclidean norm.

Although a multiplication having these properties exists in 1-space (the usual multiplication) and in 2-space (complex multiplication), it is known that a multiplication satisfying all of the above properties does not exist in any higher dimension [1]. In order to construct a multiplication in higher dimensions some properties must be sacrificed. Two examples of algebras which make such sacrifices are the quaternions in 4-space, which lack commutativity, and the Cayley numbers in 8-space, which further lack associativity.

The object of this paper is to present a multiplication which satisfies properties $\mathrm{A}, \mathrm{B}$, and $\mathrm{D}^{\prime}$. When $n+1$ is prime, this multiplication approaches satisfying $C$ and $C^{\prime}$ in a manner to be made precise below. This multiplication is of particular interest due to its applications in the areas of pattern recognition and information processing.

The paper proceeds by first constructing the algebra and its norm, then embedding it into Euclidean space.

2. The algebra. Let $R$ represent the real numbers and $Q$ the rationals. Let $R[x]$ and $Q[x]$ represent the respective polynomial rings. Define the polynomial $p_{n}$ by $p_{n}=x^{n}+x^{n-1}+\cdots+x+1$. Thus $p_{n}$ is in both $R[x]$ and $Q[x]$. Let $R_{n}$ be the quotient ring of $R[x]$ over the ideal generated by $p_{n}$ and define $Q_{n}$ similarly. From standard ring theory we know that $R_{n}$ and $Q_{n}$

Received by the editors April 18, 1977.

AMS (MOS) subject classifications (1970). Primary 13J99; Secondary 54H10, 55C99, 57A15.

Key words and phrases. Multiplication, Euclidean space, commutative algebra. 
are fields if and only if $p_{n}$ does not factor in $R[x]$ and $Q[x]$, respectively. Otherwise the quotient ring will contain zero divisors. From standard analysis we know that $p_{n}$ is prime (i.e. does not factor) in $R[x]$ and $Q[x]$ for $n=1$ or 2 and $p_{n}$ is never prime in $R[x]$ for larger $n$. A more obscure fact is that $p_{n}$ is prime in $Q[x]$ if and only if $n+1$ is prime. The "if" portion of this last statement is indicated in [2, p. 122]. The "only if" portion follows from the observation that if $n+1=a b$ then $p_{a-1}$ divides $p_{n}$. Let us summarize these remarks in a proposition.

Proposition 2.1. $R_{n}$ is a field for $n=1$ or 2 and contains zero divisors otherwise. $Q_{n}$ is a field whenever $n+1$ is prime and contains zero divisors otherwise.

It is useful to develop a simple notation for elements of $R_{n}$ and $Q_{n}$. First notice that $x^{n+1}=1+(x-1) p_{n}$. It follows that any equivalence class of polynomials corresponding to an element of $R_{n}$ or $Q_{n}$ can be represented by a polynomial of degree $n$ or less. Further, if $q_{1}$ and $q_{2}$ are any two polynomials of degree $n$ or less representing the same equivalence class then $q_{1}-q_{2}$ is a scalar multiple of $p_{n}$. We take advantage of these facts to construct a convenient notation. Specifically, we represent elements of $R_{n}$ and $Q_{n}$ as $n+1$ vectors $A=\left(a_{0}, a_{1}, \ldots, a_{n}\right)$, where $a_{i}$ is the coefficient of $x^{i}$ in any polynomial $q$ of degree $n$ or less which lies in the corresponding equivalence class. Any two such vectors $A$ and $B$ represent the same element if $A-B$ is a scalar multiple of the vector $J$ whose entries are all l's.

To formalize the above discussion, let $R_{n}^{*}\left(Q_{n}^{*}\right)$ be the set of equivalence classes of $n+1$ tuples of real (rational) numbers defined by the relation $v \sim w$ provided $v-w=k J$, where $J$ is an $n+1$ vector of l's and $k$ is a scalar. Define addition in $R_{n}^{*}$ and $Q_{n}^{*}$ by

$$
\left(a_{0}, a_{1}, \ldots, a_{n}\right)+\left(b_{0}, b_{1}, \ldots, b_{n}\right)=\left(a_{0}+b_{0}, a_{1}+b_{1}, \ldots, a_{n}+b_{n}\right)
$$

and define multiplication by

$$
\left(a_{0}, a_{1}, \ldots, a_{n}\right) *\left(b_{0}, b_{1}, \ldots, b_{n}\right)=\left(c_{0}, c_{1}, \ldots, c_{n}\right),
$$

where $c_{j}=\sum a_{s} b_{t}$, the sum being taken over all subscripts $s$ and $t$ for which $s+t \equiv j \bmod (n+1)$. Define scalar multiplication in the usual fashion. These definitions and the previous discussion yield the following proposition.

Proposition 2.2. $R_{n}^{*}\left(Q_{n}^{*}\right)$ is an algebra over the reals (rationals) isomorphic with $R_{n}\left(Q_{n}\right)$.

3. The norm. Define the function $N$ from $R_{n}^{*}$ and $Q_{n}^{*}$ to the reals by $N(A)=\left[(n+1)^{2} \operatorname{Var}(A) / n\right]^{1 / 2}$, where $\operatorname{Var}(A)$ is the sample variance of the entries of $A$. Explicitly,

$$
\operatorname{Var}(A)=[1 /(n+1)] \sum\left[a_{i}-\left[\sum a_{i} /(n+1)\right]\right]^{2},
$$

both sums being taken as $i$ goes from 0 to $n$. From the elementary fact that 
$\operatorname{Var}(b A+c)=b^{2} \operatorname{Var}(A)$ for scalars $b$ and $c$, it follows that $N$ is well defined and that $N(b A)=|b| N(A)$. Clearly $N(A) \geqslant 0$. Further, $N(A)=0$ iff $\operatorname{Var}(A)$ $=0$ iff all entries in $A$ are the same iff $A$ represents the zero element in $R_{n}^{*}$ and $Q_{n}^{*}$. A simple computation shows that $N(A)=1$ when $A=(1,0, \ldots, 0)$, the multiplicative unity in $R_{n}^{*}$ and $Q_{n}^{*}$. To show that $N$ is a norm it remains only to establish that $N(A+B) \leqslant N(A)+N(B)$. Without loss of generality, we choose both $A$ and $B$ so that the sum of their entries is zero. Under this condition the variance simplifies to

$$
\operatorname{Var}(A)=(1 /(n+1)) \sum a_{i}^{2} \text { and } N(A)=\left[(n+1) \sum a_{i}^{2} / n\right]^{1 / 2} \text {. }
$$

The following sequence of implications results:

$$
\begin{aligned}
\sum x_{i} y_{i} & \leqslant\left(\sum x_{i}^{2} \sum y_{i}^{2}\right)^{1 / 2} \quad \text { (Schwarz's inequality) } \\
& \text { iff } \sum x_{i}^{2}+\sum y_{i}^{2}+2 \sum x_{i} y_{i} \leqslant \sum x_{i}^{2}+\sum y_{i}^{2}+2\left(\sum x_{i}^{2} \sum y_{i}^{2}\right)^{1 / 2} \\
& \text { iff }\left[\sum\left(x_{i}+y_{i}\right)^{2}\right]^{1 / 2} \leqslant\left(\sum x_{i}^{2}\right)^{1 / 2}+\left(\sum y_{i}^{2}\right)^{1 / 2} \\
& \text { iff }[\operatorname{Var}(A+B)]^{1 / 2} \leqslant[\operatorname{Var}(A)]^{1 / 2}+[\operatorname{Var}(B)]^{1 / 2} \\
& \text { iff } N(A+B) \leqslant N(A)+N(B) .
\end{aligned}
$$

We summarize this discussion in a proposition.

Proposition 3.1. $N$ is a norm on $R_{n}^{*}$ and $Q_{n}^{*}$ which takes the value 1 on the multiplicative unity.

This means that $R_{n}^{*}$ and $Q_{n}^{*}$ are algebras with norms. However, they are not always normed algebras in the usual sense since this also requires the condition that $N(A * B) \leqslant N(A) N(B)$ [3]. Indeed, define $A$ in $R_{3}^{*}$ and $Q_{3}^{*}$ by $A=(1,0,1,0)$. Then it is easy to compute that $A^{2}=(2,0,2,0)$ with $N(A)=(4 / 3)^{1 / 2}$ and $N\left(A^{2}\right)=(16 / 3)^{1 / 2}$. It follows that $N\left(A^{2}\right)=2.31>$ $1.33=N(A)^{2}$.

If the above condition were to hold, the continuity of multiplication would follow immediately. Since it does not, proving this fact will require some work. To begin with we need to take a closer look at the multiplication operation in $R_{n}^{*}$ and $Q_{n}^{*}$. Recall that if $A=\left(a_{0}, \ldots, a_{n}\right)$ and $B=$ $\left(b_{0}, \ldots, b_{n}\right)$ then the $j$ th term of the produce $A * B$ is given by $\sum a_{s} b_{t}$, the sum being taken over all $s$ and $t$ for which $s+t=j \bmod (n+1)$. Define a shift operator $f$ on $n+1$ vectors by

$$
f(V)=f\left(\left(v_{0}, v_{1}, \ldots, v_{n}\right)\right)=\left(v_{n}, v_{0}, \ldots, v_{n-1}\right) .
$$

Notice that the $j$ th entry of $f(V)$ is the element of $V$ whose subscript is congruent to $j-1 \bmod (n+1)$. More generally, if $f^{k}(V)_{j}$ represents the $j$ th entry of $f^{k}(V)$, then $f^{k}(V)_{j}$ is the unique element of the original $V$ whose subscript is congruent to $j-k \bmod (n+1)$. It follows that $\sum_{k=0}^{n} a_{k} f^{k}(B)_{j}$ is the sum of all products of elements of $A$ and $B$ the sum of whose subscripts is 
congruent to $j \bmod (n+1)$. Thus $\sum_{k=0}^{n} a_{k} f^{k}(B)_{j}$ is the $j$ th entry of $A * B$. This generalizes to the following proposition.

Proposition 3.2. $A * B=\sum_{k=0}^{n} a_{k} f^{k}(B)$.

The next step is to get a bound on $\operatorname{Var}(A * B)$. Without loss of generality we assume that the sum of the entries of $B$, and hence $f^{k}(B)$, is zero. For convenience let $\Sigma_{k}$ represent $\sum_{k=0}^{n}$. Then the following string of equalities and inequalities holds.

$$
\begin{aligned}
\operatorname{Var}(A * B) & =\operatorname{Var}\left(\sum_{k} a_{k} f^{k}(B)\right) \quad \text { (Proposition 3.2) } \\
& =\sum_{j}\left(\sum_{k} a_{k} f^{k}(B)_{j}\right)^{2} /(n+1) \quad\left(\text { since } \sum_{j} f^{k}(B)_{j}=0\right) \\
& =\sum_{j}\left(\sum_{k} a_{k} f^{k}(B)_{j}\right)\left(\sum_{m} a_{m} f^{m}(B)_{j}\right) /(n+1) \\
& =\sum_{k} \sum_{m} a_{k} a_{m}\left(\sum_{j} f^{k}(B)_{j} f^{m}(B)_{j}\right) /(n+1) \\
& \leqslant \sum_{k} \sum_{m}\left|a_{k}\right|\left|a_{m}\right|\left(\sum_{j} b_{j}^{2}\right) /(n+1) \quad(\text { Schwarz's inequality) } \\
& =\left(\sum_{k}\left|a_{k}\right|\right)^{2} \operatorname{Var}(B) .
\end{aligned}
$$

It follows that $N(A * B) \leqslant N(B) \sum_{k}\left|a_{k}\right|$. Thus, multiplication by $A$ is a bounded linear operator on $R_{n}^{*}$ and $Q_{n}^{*}$ and hence is continuous. We record this fact in the next proposition.

Proposition 3.3. Multiplication is continuous in $R_{n}^{*}$ and $Q_{n}^{*}$ with respect to the norm $N$.

4. Euclidean space. In the previous sections we have constructed the algebras $R_{n}^{*}$ and $Q_{n}^{*}$ and provided them with the common norm $N$. Our goal now is to define a mapping $M$ from $R_{n}^{*}$ and $Q_{n}^{*}$ to Euclidean $n$-space, $R^{n}$. We want this mapping to have the following properties:

A. It is an injection and, in the case of $R_{n}^{*}$, is also a surjection.

B. It is a linear mapping on the underlying vector space structures.

C. It is an isometry.

Such a mapping will carry over the multiplicative structure of $R_{n}^{*}$ (and $Q_{n}^{*}$ ) into $R^{n}$ to define multiplication there.

We begin by stating a lemma which is easily proved by induction.

LEMMA 4.1. For $i=1,2, \ldots, n, \sum_{j=0}^{i-1} 1 /[(n-j)(n-j+1)]=i /[(n+$ 1) $(n-i+1)]$. 
For $i$ going from 0 to $n$, define $x_{i}$ by $x_{i}=[[(n-i)(n+1)] /[(n-i+$ 1) $n]]^{1 / 2}$. Define $v_{i}$ in $R^{n}$ by

$$
v_{i}=\left(-x_{0} / n,-x_{1} /(n-1), \ldots,-x_{i-1} /(n-i+1), x_{i}, 0, \ldots, 0\right) .
$$

Thus, $v_{i}$ has $n$ entries, the zeroth through the $n$ minus first. Of these the $i$ th is $x_{i}$, all entries before the $i$ th are of the form $-x_{j} /(n-j)$, and all entries past the $i$ th are zero. It is clear from the definition that $\sum_{i=0}^{n} v_{i}=(0,0, \ldots, 0)$ and that $v_{0}$ through $v_{n-1}$ are a basis for $R^{n}$. We will need a few other facts concerning the $v_{i}$ 's. First,

$$
\begin{aligned}
\left|v_{i}\right|^{2}= & x_{i}^{2}+\sum_{j=0}^{i-1}\left[x_{j} /(n-j)\right]^{2} \\
= & {[(n-i)(n+1)] /[(n-i+1) n] } \\
& +[(n+1) / n] \sum_{j=0}^{i-1} 1 /[(n-j)(n-j+1)] \\
= & {[(n-i)(n+1)] /[(n-i+1) n]+i /[(n-i+1) n] } \\
= & 1 .
\end{aligned}
$$

If - represents the usual dot product in $R^{n}$ then, for $i<k$,

$$
\begin{aligned}
v_{i} \cdot v_{k} & =-x_{i}^{2} /(n-i)+\sum_{j=0}^{i-1}\left[x_{j} /(n-j)\right]^{2} \\
& =-(n+1) /[(n-i+1) n]+i /[(n-i+1) n]
\end{aligned}
$$

(using Lemma 4.1 as above)

$$
-1 / n \text {. }
$$

Thus the $v_{i}$ 's are all unit vectors and all are separated by the common angle $\cos ^{-1}(-1 / n)$. This means that the $v_{i}$ 's are arranged in the same manner as the lines from the center of a standard Euclidean $n$-simplex to its $n+1$ vertices.

Define the mapping $M$ from $R_{n}^{*}$ and $Q_{n}^{*}$ to $R^{n}$ by

$$
M(A)=M\left(\left(a_{0}, a_{1}, \ldots, a_{n}\right)\right)=a_{0} v_{0}+a_{1} v_{1}+\cdots+a_{n} v_{n} .
$$

$M$ is well defined since $M(A+k J)=M(A)+k\left(\sum_{i=0}^{n} v_{i}\right)=M(A)$. To see that $M$ is an injection, notice that each element $A$ in $R_{n}^{*}$ or $Q_{n}^{*}$ has a unique representation of the form $A=\left(a_{0}, a_{1}, \ldots, a_{n-1}, 0\right)$. Recalling that $v_{0}$ through $v_{n-1}$ is a basis for $R^{n}$, it is clear that distinct elements of $R_{n}^{*}$ and $Q_{n}^{*}$ are mapped to distinct points in $R_{n}$. Further, the point $p$ in $R^{n}$ has a unique representation as $\sum_{i=0}^{n-1} p_{i} v_{i}$ and thus is the image of the element represented by $\left(p_{0}, p_{1}, \ldots, p_{n-1}, 0\right)$ in $R_{n}^{*}$. If all the $p_{i}$ 's are rational, it is also the image of the corresponding element of $Q_{n}^{*}$. That

$$
M(A+B)=M(A)+M(B) \text { and } M(k A)=k M(A)
$$


follows immediately from the distributive law in $R^{n}$. We summarize this discussion in a proposition.

Proposition 4.2. $M: R_{n}^{*} \rightarrow R^{n}$ is a vector space isomorphism. $M$ restricted to $Q_{n}^{*}$ is an injection onto a dense subset of $R^{n}$, namely those points which can be expressed as a rational linear combination over the basis $v_{0}, v_{1}, \ldots, v_{n-1}$. (Caution: These are not the points which have rational coefficients over the usual basis.)

We next check that $M$ is norm preserving. Express $M(A)$ in terms of the usual basis in $R^{n}$ as $M(A)=\left(w_{0}, w_{1}, \ldots, w_{n-1}\right)$. From the definitions of $v_{i}$ and $x_{i}$ it follows that $w_{i}=x_{i}\left[a_{i}-\sum_{j=i+1}^{n} a_{j} /(n-i)\right]$. Then,

$$
\begin{aligned}
|M(A)|^{2} & =\sum_{i=0}^{n-1} w_{i}^{2}=\sum_{i=0}^{n-1} x_{i}^{2}\left[a_{i}-\sum_{j=i+1}^{n} a_{j} /(n-i)\right]^{2} \\
& =[(n+1) / n] \sum_{i=0}^{n-1}\left[(n-i) a_{i}-\sum_{j=i+1}^{n} a_{j}\right]^{2} /[(n-i+1)(n-i)] .
\end{aligned}
$$

To simplify this last expression, let us first observe that it contains only quadratic terms in the $a_{i}$ 's when in its expanded form. So all we need to know are the coefficients $c_{i}$ of $a_{i}^{2}$ and $c_{i k}$ of $a_{i} a_{k}(i<k)$. Note that $a_{i}$ will not appear in any terms of the sum past the $i$ th, that it has a distinguished role in the $i$ th term, and that it has a standard role in terms preceeding the $i$ th. In particular, the contribution to $c_{i}$ from the $i$ th term is

$$
[(n+1) / n]\left[(n-i)^{2} /[(n-i+1)(n-i)]\right]
$$

and the contribution from previous terms is

$$
[(n+1) / n] \sum_{j=0}^{i-1} 1 /[(n-j+1)(n-j)] .
$$

Therefore,

$$
\begin{aligned}
c_{i} & =[(n+1) / n]\left[(n-i) /(n-i+1)+\sum_{j=0}^{i-1} 1 /[(n-j+1)(n-j)]\right] \\
& =[(n+1) / n][(n-i) /(n-i+1)+i /[(n+1)(n-i+1)]] \\
& \quad(\text { Lemma 4.1) } \\
& =1 .
\end{aligned}
$$

Similarly, the contribution to $c_{i k}$ from the $i$ th term is

$$
[(n+1) / n][-2(n-i) /[(n-i+1)(n-i)]]
$$

and the contribution from the previous terms is

$$
[(n+1) / n] \sum_{j=0}^{i-1} 2 /[(n-j+1)(n-j)] \text {. }
$$


Expanding as above one finds that $c_{i k}=-2 / n$. It follows that $|M(A)|^{2}=$ $\sum_{i=0}^{n} a_{i}^{2}-2 \sum_{i<k} a_{i} a_{k} / n$. However,

$$
\begin{aligned}
N(A)^{2} & =\left[(n+1)^{2} / n\right] \operatorname{Var}(A) \\
& =[(n+1) / n] \sum_{i=0}^{n}\left[a_{i}-\sum_{j=0}^{n} a_{j} /(n+1)\right]^{2} \\
& =[(n+1) / n] \sum_{i=0}^{n}\left(n a_{i}-\sum_{k \neq i} a_{k}\right)^{2} /(n+1)^{2} .
\end{aligned}
$$

Again, observe that this expression contains only quadratic terms in the $a_{i}$ 's when expanded. Let $c_{i}^{\prime}$ be the coefficient of $a_{i}^{2}$ and $c_{i k}^{\prime}$ be the coefficient of $a_{i} a_{k}(i<k)$ in this expansion. It is easy to see that $c_{i}^{\prime}=[1 /[n(n+1)]]\left(n^{2}+n\right)$ $=1$ and $c_{i k}^{\prime}=[1 /[n(n+1)]][-4 n+2(n-1)]=-2 / n$. Therefore,

$$
N(A)^{2}=\sum_{i=0}^{n} a_{i}^{2}-2 \sum_{i<k} a_{i} a_{k} / n=|M(A)|^{2} .
$$

This proves the following proposition.

Proposition 4.3. The mapping $M$ from $R_{n}^{*}$ or $Q_{n}^{*}$ to $R^{n}$ is an isometry.

We can now define multiplication in $R^{n}$ by simply carrying over the multiplication in $R_{n}^{*}$. For $p$ and $q$ in $R^{n}$ define $p^{*} q$ by $p^{*} q=$ $M\left(M^{-1}(p)^{*} M^{-1}(q)\right)$. Since $M$ is an isometry and a vector space isomorphism, all the metric and algebraic properties of the multiplication are preserved. We record this fact in the following theorem.

THEOREM 4.4. The operation ${ }^{*}$ defined on $R^{n}$ together with the usual vector space structure makes $R^{n}$ into a commutative, associative algebra with multiplicative unity $(1,0, \ldots, 0)$. This multiplication is continuous in the Euclidean metric. This algebra restricted to the dense subset of $R^{n}$ which is the image of $Q_{n}^{*}$ under $M$ is a division algebra iff $n+1$ is prime.

To get more insight into how this multiplication works, let us compute the product of the two points $(a, b)$ and $(c, d)$ in $R^{2}$. In order to take $M^{-1}$ of these points we first express them in terms of the basis $v_{0}, v_{1}$ of $R^{2}$. These are easily seen to be $v_{0}=(1,0)$ and $v_{1}=\left(-1 / 2,3^{1 / 2} / 2\right)$. Thus

$$
(a, b)=\left(a+b / 3^{1 / 2}\right) v_{0}+\left(2 b / 3^{1 / 2}\right) v_{1}
$$

while

$$
(c, d)=\left(c+d / 3^{1 / 2}\right) v_{0}+\left(2 d / 3^{1 / 2}\right) v_{1}
$$

Then

$$
M^{-1}((a, b))=\left(a+b / 3^{1 / 2}, 2 b / 3^{1 / 2}, 0\right)
$$

and

$$
M^{-1}((c, d))=\left(c+d / 3^{1 / 2}, 2 d / 3^{1 / 2}, 0\right) .
$$


Using the product formula in $R_{2}^{*}$ we find

$$
\begin{aligned}
& M^{-1}((a, b))^{*} M^{-1}((c, d)) \\
& \quad=\left(a c+b d / 3+(a d+b c) / 3^{1 / 2}, 4 b d / 3+2(a d+b c) / 3^{1 / 2}, 4 b d / 3\right) \\
& =\left(a c-b d+(a d+b c) / 3^{1 / 2}, 2(a d+b c) / 3^{1 / 2}, 0\right),
\end{aligned}
$$

which maps to $(a c-b d, a d+b c)$ in $R^{2}$. It follows that $R^{2}$ endowed with this multiplication is isomorphic with the field of complex numbers. Similar computations show that the one dimensional subspace of $R^{n}$ consisting of those elements of the form $(x, 0, \ldots, 0)$ is a subalgebra isomorphic to the field of real numbers.

Another interesting subalgebra can be obtained by taking the image in $R^{n}$ of all those elements of $Q_{n}^{*}$ which have a representation in which all the entries are integers. It is easily seen to be a subalgebra. It turns out that these points form a regular array in $R^{n}$. Indeed, the region of $R^{n}$ consisting of those points closest to a given point in the array has the form of an $n+1$ permutahedron. This is an $n$ dimensional convex figure whose vertices correspond to the permutations of $n+1$, with two vertices being adjacent whenever they differ by an elementary transposition. This subalgebra therefore provides an algebraic structure to the permutahedron packing of $n$-space.

\section{REFERENCES}

1. W. B. Gordon, An application of Hadamard's inverse function theorem to algebra, M.A.A. Monthly 84 (1977), 28.

2. I. N. Herstein, Topics in algebra, Blaisdell, Waltham, Mass., 1964.

3. M. A. Naimark, Normed rings, Noordhoff, Groningen, 1959.

Martin Marietta Aerospace, P. O. Box 179, Denver, Colorado 80201 